\title{
Updates in drug-induced acute pancreatitis
}

\author{
Omkolsoum Alhaddad ${ }^{1}$, Maha Elsabaawy ${ }^{1 *}$ (D), Marwa Elfauomy ${ }^{1}$, Dalia Elsabaawy ${ }^{2}$ and Tarek Mansour $^{3}$
}

\begin{abstract}
Background: Being infrequent, drug-induced acute pancreatitis (DIP) is an overlooked clinical entity that can be serious with significant morbidity and mortality.

Main body: A renovative review of drugs incriminated in acute pancreatitis had been presented with all relevant data and case presentations. Antibiotics, antidiabetics, antihypertensive agents, H2 blockers (H2B) and proton pump inhibitors (PPIs), anticancer therapies, and the new direct-acting antiviral therapies (DAAs) of hepatitis C virus (HCV) were discussed pertinently to DIP.
\end{abstract}

Conclusions: DIP should be suspected as a potential adverse event to every newly emerged drug. Herein, an updated review of drugs recently alleged to be implicated in DIP.

\section{Background}

\section{Prevalence of DIP}

Drug-induced pancreatitis, a potentially forgotten diagnosis that should be rigorously appreciated. In fact, the increasing number of pharmacological agents is associated with increased incidences of acute pancreatitis. Drug-induced pancreatitis represents up to $5 \%$ of all acute pancreatitis cases, hence, can be considered as the third common cause of acute pancreatitis after alcohol and gall stones are ruled out [1]. The worldwide incidence of drug-induced pancreatitis is spanning around $5-80$ per 100,000 adults. DIP is reported to be higher in patients with inflammatory bowel disease, also, in children, geriatric population, and with immunosuppression [2].

Currently, more than 500 drugs have been reported in the World Health Organization database as offenders of acute pancreatitis [3].

\section{Diagnostic concerns of DIP}

Discerning a diagnosis of DIP has neither clinical nor laboratory diagnostic criteria. However, the diagnosis of acute pancreatitis is clinically persuaded through disappearance of abdominal pain and drop of pancreatic

\footnotetext{
* Correspondence: maha.ahmed@liver.menofia.edu.eg

1 Department of Hepatology and Gastroenterology, National Liver Institute,

Menoufia University, Shebeen El-Kom, Menoufia 32511, Egypt

Full list of author information is available at the end of the article
}

enzymes upon drug discontinuation, exclusion of other common etiologies, and recurred manifestations upon challenging the suspected drug [4]. Re-challenging the suspected drug is not feasible in critical patients; however, being applied will reduce the number of culprit medications. Exclusion of uncommon etiologies and other possible drugs than the drug of concern in multimedicated patients is always difficult.

Badalov classification system of drug-induced acute pancreatitis contains 5 classes: Ia, Ib, II, III, and IV. The number of published cases, re-challenge, and latency provides the basis of Badalov classification (Table 1) [5].

The latency between initiation of the offending drug and developing acute pancreatitis can be short (less than 24h), intermediate (1-30 days), and long (more than 30 days). According to Badalov, the latency is described as consistent with the drug-related pancreatitis when $75 \%$ of cases were related to any of the previously mentioned groups [5].

\section{Review of potential offenders Propofol}

Propofol introduced itself to the market about three decades ago as a safer short-acting anesthetic agent. Its use is extended as long-term hypnotic and sedative infusion in critically ill patients. Significant weight of publications and case reports mentioned a causal relationship between propofol and acute pancreatitis [6]. A recent

\section{Springer Open}

(c) The Author(s). 2020 Open Access This article is licensed under a Creative Commons Attribution 4.0 International License, which permits use, sharing, adaptation, distribution and reproduction in any medium or format, as long as you give appropriate credit to the original author(s) and the source, provide a link to the Creative Commons licence, and indicate if changes were made. The images or other third party material in this article are included in the article's Creative Commons licence, unless indicated otherwise in a credit line to the material. If material is not included in the article's Creative Commons licence and your intended use is not permitted by statutory regulation or exceeds the permitted use, you will need to obtain permission directly from the copyright holder. To view a copy of this licence, visit http://creativecommons.org/licenses/by/4.0/. 
Table 1 Badalov classification system of drug-induced acute pancreatitis [5]

\begin{tabular}{ll}
\hline Class la drugs & At least 1 case report with positive re-challenge, excluding all other causes, such as alcohol, hypertriglyceridemia, \\
& gallstones, and other drugs \\
Class Ib drugs & At least 1 case report with positive re-challenge, however, other causes, such as alcohol, hypertriglyceridemia, \\
& gallstones, and other drugs were not ruled out \\
Class II drugs & At least 4 cases in the literature and consistent latency in at least $75 \%$ of cases \\
Class III drugs & At least 2 cases in the literature with neither consistent latency among cases nor re-challenge \\
Class IV drugs & Like class III drugs, but only one case report had been published, without re-challenge \\
\hline
\end{tabular}

systematic review specified the propofol-pancreatitis relationship as probable [7]. According to Badalov et al. classification system, propofol is a class Ia drug as acute pancreatitis is documented along with positive drug rechallenge [7]. Propofol is suspended in a lipid emulsion that can be a factor in propofol-related hypertriglyceridemia in susceptible people. Propofol-related pancreatitis has been attributed to developed hypertriglyceridemia, a traditional etiology for acute pancreatitis [8]. Hypertriglyceridemia more than $1000 \mathrm{mg} / \mathrm{dl}$ was documented at time of acute pancreatitis in reports of propofol-pancreatitis; however, in other propofol, pancreatitis was documented in absence of hypertriglyceridemia [9]. Hence, acute pancreatitis inferred to propofol could have many mechanisms. Intensive care unit (ICU) patients and prolonged high rate propofol administration are considered risk factors for propofolpancreatitis [10]. Accordingly, monitoring serum triglycerides, amylase, and lipase is advisable in cases on prolonged propofol administration. Acute pancreatitis should be suspected in any patient developing abdominal pain after propofol infusion and the drug should be discontinued [10]. Yet, this area of propofol-associated acute pancreatitis is considered as uncertain.

\section{Antibiotics}

Minocycline is a tetracycline while tigecycline is a glycylcycline and a structural derivative of minocycline [11]. Tigecycline shares similar pharmacokinetic properties and adverse effects with tetracyclines. Acute pancreatitis tightly linked to tetracycline was not listed in FDA approval of either minocycline or tigecycline [12].

Minocycline is widely used for treatment of acne vulgaris. Also indicated for methicillin-resistant Staphylococcus aureus (MRSA)-related infections and rheumatoid arthritis as a disease-modifying drug [13]. A small case series described minocycline-induced pancreatitis in two cystic fibrosis patients in 2001 [14]. The inherent pancreatic insufficiency in cystic fibrosis patients could play a contribution in this serious event. In these 2 cases, neither re-challenge nor consistent latency were existing and accordingly minocycline is specified as class III drug in Badalov classification for druginduced acute pancreatitis [14]. A newer two cases of minocycline-induced pancreatitis in non-cystic fibrosis patients had been recently published [15]. In both cases, all other etiologies of acute pancreatitis have been excluded and resolution of the condition was achieved few days after minocycline discontinuation [15]. Drug idiosyncrasy could explain minocycline pancreatitis in non-cystic fibrosis cases paralleling the explanation of minocycline-induced hepatotoxicity. Acute pancreatitis is a serious condition and clinicians should be aware of such minocycline-related adverse event. Currently, no identifiable risk factors for minocycline-induced pancreatitis.

Few case reports linked tigecycline to acute pancreatitis with variable time latency. In these cases, tigecycline was used to treat soft tissue infections except in a case of respiratory infection in a cystic fibrosis patient [16]. Tigecycline discontinuation was followed by regression of symptoms and drop of pancreatic enzymes [16]. The number of reported tigecycline pancreatitis is small and insufficient to lower its prescriptions [17]. Concerning tigecycline pancreatitis, the retrospective analyses of phases 3 and 4 clinical studies revealed mixed conclusion. In respect to seriousness of acute pancreatitis, clinicians should be aware of this potential adverse effect and should monitor patients for features of pancreatitis during treatment with tigecycline [17].

\section{Antidiabetic drugs}

In 2008 and in response to 30 post-marketing reports, FDA has ordered a warning label about a potential risk of acute pancreatitis in patients taking exenatide and sitagliptin, the first members of incretin-based therapies [18]. Afterward, the FDA adverse drug events system received a large number of spontaneously reported cases of acute pancreatitis in those taking various incretinbased therapies [18]. Further, observational studies have raised questions as to the possible association of treatment with incretin-based therapies, particularly with DPP-4 inhibitors and the risk of acute pancreatitis [19]. In 2016, in JAMA, a large multicenter and populationbased study had indicated a non-significant increased risk of incretin-associated acute pancreatitis compared to other antidiabetics [20]. Also, the separate secondary analyses for glucagon-like peptide-1 (GLP1) agonists and 
dipeptidyl peptidase-4 (DDP4) inhibitors showed similar results.

Sodium-glucose cotransporter-2 inhibitors (SGLT2 inhibitors), a newly approved antidiabetics, have non-beta cell-dependent mechanism of action. They inhibit SGLT2 in the proximal renal tubules and thus preventing reabsorption of filtered glucose [21]. Since approval, the literature received a handful of medical articles about a link between SGLT2 inhibitors, particularly, canagliflozin and acute pancreatitis. In 2016, FDA wrote in its website that there is a safety issue but did not mention about a causal relationship between SGLT2 inhibitors and pancreatitis. In 2018, Canada Health submitted a review investigating this issue. The review concluded that there may be a link between SGLT2 inhibitors and acute pancreatitis, and physicians should be vigilant to symptoms of acute pancreatitis in patients taking these drugs [22].

\section{Antihypertensive agents}

Case reports of acute pancreatitis induced by enalapril, lisinopril, captopril, ramipril, and perindopril have been published. Some of the published reports mentioned acute pancreatitis after lisinopril re-challenge. The proposed mechanism is pancreatic angioedema with pancreatic duct obstruction [23]. A recent population-based study found that, in absence of alcohol, 7 per 10,000 attacks of acute pancreatitis/year are among users of angiotensin-converting enzyme (ACE) inhibitors [23].

Angiotensin receptor blockers (ARBs) induced pancreatitis have been introduced in a few case reports denoting a lesser risk of pancreatitis [24].

\section{Acid-suppressing drugs}

In many case reports, cimetidine and ranitidine have been associated with acute pancreatitis. Of note, acidsuppressing drugs can be prescribed for a prodrome of acute pancreatitis [25]. It was determined that hypergastrinemia induced by continuous use of proton pump inhibitors (PPIs) can stimulate pancreatic enzyme secretion eventually resulting in pancreatitis [26]. The literature contains several case reports of omeprazole-, pantoprazole-, and lansoprazole-induced pancreatitis. In more than one report, the pancreatitis was severe and necrotizing. In rats, intraperitoneal infusion of omeprazole resulted in pancreatic inflammation and elevated pancreatic enzymes. According to Badalov classification, proton pump inhibitors are specified as class I drugs based on positive re-challenge with the drug and consistent latency [27].

\section{Anticancer therapies}

Anticancer drugs almost always carry the risk of precipitation acute pancreatitis. Many of the older and recently approved anticancer therapies have been listed to cause pancreatitis. From the older agents, asparaginase used for the treatment of acute lymphoblastic leukemia is the most frequently chemotherapeutic agent-causing pancreatitis [28]. Asparaginase-related pancreatitis had been reported at an incidence of $2 \%$ to $16 \%$. Asparaginase pancreatitis is severe in up to $15 \%$ of patients and positive re-challenge is reported in up to $60 \%$ of cases [28]. Cisplatin and oxaliplatin are listed as inducing pancreatitis and discontinuation of these drugs had showed regression of symptoms and laboratory evidence of pancreatitis [29]. Everolimus and tamoxifen cause elevated serum triglycerides and hypertriglyceridemia can be the underlying mechanism of reported cases of everolimus and tamoxifen pancreatitis [30]. In many case reports and small case series, newer anticancer agents, immune check point inhibitors, tyrosine kinase inhibitors, and proteasome inhibitors have been reported to cause acute pancreatitis [31]. The proposed mechanisms of pancreatitis are immune-mediation direct toxicity or hypertriglyceridemia [31]. In concern to immune check inhibitors, nivolumab- and pembrolizumab-related pancreatitis have been recently reported in $1.8 \%$ of patients. Also, ipilimumab approved for treatment of melanoma is reported to be associated with pancreatitis in less than $1 \%$ of cases.

Pancreatitis attributed to check point inhibitors is considered as immune toxicities and in all cases, corticosteroids were the therapeutic option. The older tyrosine kinase inhibitors (TKIs), sorafenib, sunitinib, and imatinib are reported to cause pancreatitis [32]. Recent case reports have linked the newer tyrosine kinase inhibitors (TKIs), vemurafenib and ponatinib to pancreatitis and just elevated pancreatic enzymes. The famous proteasome inhibitor, bortezomib, has been repeatedly reported to cause pancreatitis [33].

Nonselective trans-arterial chemoembolization (TACE) may lead to elevated amylase in up to $40 \%$ of cases, while TACE-related pancreatitis can happen in up to $4 \%$ of cases. The larger the area infused with the chemotherapeutic agent, the greater the risk of pancreatitis [34]. Post-TACE-necrotizing pancreatitis is thought to be ischemic in nature secondary to embolic blocking of the pancreatic blood supply [35].

\section{The new direct-acting antiviral therapies (DAAs) of hepatitis $\mathrm{C}$ virus $(\mathrm{HCV})$}

DIP occurrence with the previously known combination of peginterferon and ribavirin had been substantiated [36]. Nevertheless, determination whether it is interferon or ribavirin-related effect is yet uncovered as all reported cases were on combination therapy. As both drugs are immune interfering, DIP might be an expected consequence. 
Recently, the high safety profiles associating DAAs had denied any linkage with DIP occurrence. However, boceprevir and telaprevir were mentioned in such a fatal condition in two separate reports $[37,38]$. Conversely, later generations of DAAs had been mentioned to cause DIP only in cases with ribavirin including regimens. In a 75-year-old male presenting with acute pancreatitis while on 3 weeks ombitasvir, paritaprevir, ritonavir, and dasabuvir (OTV/PTV/RTV/DSV) and ribavirin $1200 \mathrm{mg}$ daily, a shift to ledipasvir/sofosbuvir regimen had improve the condition [36]. Consequently, ribavirin was principally convicted in most cases of $\mathrm{HCV}$ therapeutics-induced pancreatitis. Data are still limited on the potential role and pathogenic mechanisms of DAAs as DIP offenders.

\section{Conclusion}

Literature has recently liberated several reports of case reports concerning DIP with an array of newly emerged drugs like propofol infusion, antidiabetics (GLP1 agonists and DDP4 inhibitors, and SGLT2 inhibitors, particularly, canagliflozin), antibiotics (minocycline), antihypertensive agents (enalapril, lisinopril, captopril, ramipril, perindopril, and less frequently angiotensin receptor blockers), proton pump inhibitors (omeprazole, pantoprazole, and lansoprazole), anticancer drugs (nivolumab, pembrolizumab, ipilimumab), the newer TKIs (vemurafenib and ponatinib), proteasome inhibitors (bortezomib), and non-selective TACE, and direct-acting antiviral therapies (DAAs) of hepatitis $\mathrm{C}$ virus along with ribavirin.

DIP, which is an overlooked diagnosis in the clinical setting, must be rigorously considered whenever patients are on a set of new drugs and presenting with even, minimal symptoms and signs of acute pancreatitis. Indeed, updated vigilant clinicians and critical care medical staff can save lives.

\section{Abbreviations}

DIP: Drug-induced acute pancreatitis; ICU: Intensive care unit; FDA: Food and Drug Association; MERSA: Methicillin-resistant Staphylococcus aureus; DDP4: Dipeptidyl peptidase-4; GLP1: Glucagon-like peptide-1;

SGLT2: Sodium-glucose cotransporter-2; ACEl: Angiotensin-converting enzyme inhibitors; ARBS: Angiotensin receptor blockers; H2B: H2 blockers; PPIs: Proton pump inhibitors; TKls: Tyrosine kinase inhibitors; TACE: Transarterial chemoembolization; DAAs: Direct-acting antiviral therapies; HCV: Hepatitis C virus; OTV/PTV/RTV/DSV: Ombitasvir, paritaprevir, ritonavir, and dasabuvir

\section{Acknowledgements}

All respect and indebtedness to the great professor Dr. Omkolsoum Alhaddad who created this work for her unlimited, sincere, and priceless inputs complying this work to be fulfilled.

\section{Authors' contributions}

O A.: conceived and designed the study, analyzed and wrote the paper, M E.: analyzed the data and contributed in writing the paper. M E.: clinical evaluation and data collection. D E.: pharmacologic analysis of the data and revised the paper. T M.: revised and approved the paper. All authors have read and approved the manuscript.

\section{Funding}

This review and the publication were completely funded by all authors.

\section{Availability of data and materials}

Data used to support the findings of this study are included within the article.

\section{Ethics approval and consent to participate}

The review was conformed to the ethical guidelines of the 1975 Declaration of Helsinki and was approved by the institutional review board of the National Liver Institute (NLI IRB 00003413), Menoufia University.

\section{Consent for publication}

Not applicable for review article.

\section{Competing interests}

The authors declare that they have no competing interests.

\section{Author details}

${ }^{1}$ Department of Hepatology and Gastroenterology, National Liver Institute, Menoufia University, Shebeen El-Kom, Menoufia 32511, Egypt. ${ }^{2}$ Department of Clinical Pharmacy, National Liver Institute, Menoufia University, Menoufia 32511, Egypt. ${ }^{3}$ Department of Internal Medicine, Faculty of Medicine, Ain

Shams University, Cairo 3434, Egypt.

Received: 10 June 2020 Accepted: 12 October 2020

Published online: 19 October 2020

\section{References}

1. Scott T (2014) Drug induced acute pancreatitis: does it exist? World J Gastroenterol 20(44):16529-16534

2. Yokoe M, Takada T, Mayumi T, Yoshida M, Isaji S, Wada K et al (2015) Japanese guidelines for the management of acute pancreatitis: Japanese guidelines 2015. J Hepatobiliary Pancreat Sci 22(6):405-432

3. Rekhi B, Mannem S, Rekhi H, Mittal S, Arora S, Sathya P et al (2017) A case report on drug induced pancreatitis due to levofloxacin and methylprednisolone. Int J Case Rep Images 8(1):62-65

4. Ghatak R, Masso L, Kapadia D, Kulairi Z (2017) Medication as a cause of acute pancreatitis. Am J Case Rep 18:839-841

5. Badalov N, Baradarian R, Iswara K, Li J, Steinberg W, Tenner S (2007) Druginduced acute pancreatitis: an evidence-based review. Clin Gastroenterol Hepatol 5:648-661

6. Jones M, Hall O, Kaye AM, Kaye AD (2015) Drug-induced acute pancreatitis: a review. Ochsner J 15:45-51

7. Asghar MU, Cheema HA, Tanveer K, Leinwand J (2019) Propofol infusion and acute pancreatitis: a review. Am J Ther 28

8. Kaur H, Nattanamai P, Qualls KE (2018) Propofol and clevidipine-induced hypertriglyceridemia. Cureus. 10(8):e3165

9. Haffar S, Kaur RJ, Garg SK et al (2019) Acute pancreatitis associated with intravenous administration of propofol: evaluation of causality in a systematic review of the literature. Gastroenterol Rep (Oxf) 7(1):13-23

10. Devlin JW, Lau AK, Tanios MA (2005 Oct) Propofol-associated hypertriglyceridemia and pancreatitis in the intensive care unit: an analysis of frequency and risk factors. Pharmacotherapy 25(10):1348-1352

11. Greer ND (2006) Tigecycline (Tygacil): the first in the glycylcycline class of antibiotics. Proc (Bayl Univ Med Cent) 19(2):155-161

12. Lin J, Wang R, Chen J (2018) Tigecycline-induced acute pancreatitis in a renal transplant patient: a case report and literature review. BMC Infect Dis 18(1):201

13. Alexis A, Del Rosso JQ, Desai SR et al (2018) BPX-01 minocycline topical gel shows promise for the treatment of moderate-to-severe inflammatory acne vulgaris. J Clin Aesthet Dermatol 11(11):25-35

14. Boyle MP (2001) Minocycline-induced pancreatitis in cystic fibrosis. Chest. 119(4):1283-1285

15. Gabriel JG, Bhogal S, Kapila A Minocycline associated pancreatitis. Am J Ther $2017 ; 0,1-2$. 
16. Hung WY, Kogelman L, Volpe G, lafrati M, Davidson L (2009) Tigecyclineinduced acute pancreatitis: case report and literature review. Int J Antimicrob Agents 34(5):486-489

17. Akhter S, Krishnan P, Kaul P.Tigecycline-associated acute pancreatitis. Am J Ther 2018 0, 1-2.

18. U.S. Food and Drug Administration MedWatch. MedWatch safety alerts for drugs, biologics, medical devices, and dietary supplements. 2008; 8;2012.

19. Thomsen R, Pedersen L, Møller N, Kahlert J, Beck-Nielsen H, Sørensen H (2015) Incretin-based therapy and risk of acute pancreatitis: a nationwide population-based case-control study. Diabetes Care 38(6):1089-1098

20. Azoulay L, Filion KB, Platt RW et al (2016) Association between incretinbased drugs and the risk of acute pancreatitis. JAMA Intern Med 176(10): 1464-1473

21. Gutch M, Bhattacharya A, Kumar S, Pahan R, Singh R (2018) Dapagliflozin induced pancreatitis. Int J Med Public Health 8:1,45-1,47

22. Hsia DS, Grove O, Cefalu WT (2017) An update on sodium-glucose cotransporter-2 inhibitors for the treatment of diabetes mellitus. Curr Opin Endocrinol Diabetes Obes 24(1):73-79

23. Thumma S, Errico K, Manchala V, Mattana J. Lisinopril-induced acute pancreatitis. Am J Ther 2018: 0, 1-4.

24. Bexelius TS, Ljung R, Mattsson F, Lu Y, Lindblad M (2017) Angiotensin II receptor blockers and risk of acute pancreatitis - a population based casecontrol study in Sweden. BMC Gastroenterol 17(1):36

25. Eland IA, Alvarez CH, Stricker BH, Rodríguez LA (2000) The risk of acute pancreatitis associated with acid-suppressing drugs. Br J Clin Pharmacol 49(5):473-478

26. McCarthy DM (2020) Proton pump inhibitor use, hypergastrinemia, and gastric carcinoids-what is the relationship? Int J Mol Sci 21(2):662

27. Murtaza G, Khalid M (2018) And Nicolas a Mungo. Recurrent pantoprazoleassociated pancreatitis. Am J Ther 25(4):e492-e493

28. Stefanović M, Jazbec J, Lindgren F, Bulajić M, Löhr M (2016) Acute pancreatitis as a complication of childhood cancer treatment. Cancer Med 5(5):827-836

29. Butt W, Saadati H, Saif M (2010) Oxaliplatin-induced pancreatitis: a case series. Anticancer Res December 30(12):5113-5115

30. Singh HK, Prasad MS, Kandasamy AK, Dharanipragada K (2016) Tamoxifeninduced hypertriglyceridemia causing acute pancreatitis. J Pharmacol Pharmacother 7(1):38-40. https://doi.org/10.4103/0976-500X.179365

31. Clamon G (2017) Pancreatitis associated with newer classes of antineoplastic therapies. JCSO 15(3):e135-e141

32. Bajwa R, Cheema A, Khan T et al (2019) Adverse effects of immune checkpoint inhibitors (programmed death-1 inhibitors and cytotoxic Tlymphocyte-associated protein-4 inhibitors): results of a retrospective study. J Clin Med Res 11(4):225-236

33. Brulc E, Seehaus C, Schutz N, Fantl D (2018) Management of pancreatitis related to Bortezomib treatment: report of two cases. Hematol Transfus Cell Ther 40(4):382-384

34. Krishnamurthy P, Brown M, Agrawal S, Short RF (2017) Acute pancreatitis as a complication of trans-arterial chemoembolization of hepatocellular cancer-case report and review of literature. J Gastrointest Oncol 8(1):E26-E30

35. Bae S, Yeon J, Lee J, Kim J, Lee H, Lee S et al (2012) A case of necrotizing pancreatitis subsequent to transcatheter arterial chemoembolization in a patient with hepatocellular carcinoma. Clin Mol Hepatol 18(3):321-325

36. Cristina C, Diaz A, Bolaños A et al DI-031 acute pancreatitis and hyperbilirubinaemia possibly associated with ribavirin administration and new direct antiviral agents. European journal of hospital pharmacy (2016); 23: A131. C.

37. Ventura R, Urich S, Skinner S et al (2013) First report of telaprevir-induced pancreatitis. Dig Dis Sci 58:887-888

38. Bilar J, Carvalho-Filho R, Mota C et al (2014) Acute pancreatitis associated with boceprevir: a case report. Braz J Infect Dis 18(4):454-456

\section{Publisher's Note}

Springer Nature remains neutral with regard to jurisdictional claims in published maps and institutional affiliations.

\section{Submit your manuscript to a SpringerOpen ${ }^{\circ}$ journal and benefit from:}

- Convenient online submission

- Rigorous peer review

- Open access: articles freely available online

High visibility within the field

- Retaining the copyright to your article

Submit your next manuscript at $\boldsymbol{\nabla}$ springeropen.com 